Bull. Austral. Math. Soc.

VOL. 65 (2002) [115-120]

\title{
A NOTE ON THE HEAT KERNEL ON THE HEISENBERG GROUP
}

\section{Adam Sikora and Jacek Zienkiewicz}

We describe the analytic continuation of the heat kernel on the Heisenberg group $\mathbb{H}_{n}(\mathbb{R})$. As a consequence, we show that the convolution kernel corresponding to the Schrödinger operator $e^{i s L}$ is a smooth function on $\mathbb{H}_{n}(\mathbb{R}) \backslash S_{s}$, where $S_{s}=\left\{(0,0, \pm s k) \in \mathbb{H}_{n}(\mathbb{R}): k=n, n+2, n+4, \ldots\right\}$. At every point of $S_{s}$ the convolution kernel of $e^{i s L}$ has a singularity of Calderón-Zygmund type.

Let $\mathbb{H}_{n}(\mathbb{R})$ denote the $(2 n+1)$-dimensional real Heisenberg group, that is, $\mathbb{R}^{n} \times \mathbb{R}^{n} \times \mathbb{R}$ with the group law

$$
(x, y, t)\left(x^{\prime}, y^{\prime}, t^{\prime}\right)=\left(x+x^{\prime}, y+y^{\prime}, t+t^{\prime}-\frac{1}{2} \operatorname{Im} \sum_{r=1}^{n}\left(x_{r}+i y_{r}\right)\left(x_{r}^{\prime}-i y_{r}^{\prime}\right)\right)
$$

for all $x, x^{\prime}, y, y^{\prime}$ in $\mathbb{R}^{n}$ and $t$ and $t^{\prime}$ in $\mathbb{R}$. For $s$ in $\mathbb{R}$, we define the set $S_{s}$ by

$$
S_{s}=\left\{(0,0, \pm s k) \in \mathbb{H}_{n}(\mathbb{R}): k=n, n+2, n+4, \ldots\right\} .
$$

We define $\mathbb{H}_{n}(\mathbb{C})$ like $\mathbb{H}_{n}(\mathbb{R})$. For $(x, y, t) \in \mathbb{H}_{n}(\mathbb{C})$, we write $x^{2}$ for $\sum_{r=1}^{n} x_{r}^{2}$, and $A$ and $B$ for it $-\left(x^{2}+y^{2}\right) / 4$ and $-\left(x^{2}+y^{2}\right) / 2$ respectively.

The vector fields $X_{r}, Y_{r}$ (where $r=1, \ldots, n$ ) and $T$, given by

$$
X_{r}=\frac{\partial}{\partial x_{r}}-\frac{y_{r}}{2} \frac{\partial}{\partial t}, \quad Y_{r}=\frac{\partial}{\partial y_{r}}+\frac{x_{r}}{2} \frac{\partial}{\partial t} \quad \text { and } \quad T=\frac{\partial}{\partial t}
$$

form a basis for the Lie algebra of left-invariant vector fields on $\mathbb{H}_{n}(\mathbb{R})$. The Heisenberg Laplacian $L$ is defined by

$$
L=-\sum_{r=1}^{n} X_{r}^{2}+Y_{r}^{2}
$$

The subelliptic operator $L$ admits a spectral resolution

$$
L=\int_{0}^{\infty} \lambda \mathrm{d} E(\lambda)
$$

Received 18th June, 2001

The research for this paper was supported by the Australian National University, Wroclaw University, and the Polish Research Council KBN (KBN 2P03A 058 14).

Copyright Clearance Centre, Inc. Serial-fee code: 0004-9727/02 \$A2.00+0.00. 
and therefore when Re $s \geqslant 0$, one can define the operator $e^{-s L}$, bounded on $L^{2}\left(\mathbb{H}_{n}(\mathbb{R})\right)$, by the spectral theorem:

$$
e^{-s L}=\int_{0}^{\infty} e^{-s \lambda} \mathrm{d} E(\lambda)
$$

Let $p_{s}$ be the convolution kernel of the operator $e^{-s L}$ (see $[5,(1.10),(1.11)]$ ). When $s>0, e^{-s L}$ is the solution operator for the Heisenberg heat equation $\partial_{s} u=-L u$ and $p_{s}$ is called the heat kernel (see [6, (7.30), p.71].

The goal of this note is to study the analytic continuation of the heat kernel $p_{s}$. This is interesting from the point of view of the theory of analytic hypoellipticity (see $[1,2])$. Another reason to study the analytic continuation of $p_{s}$ is to investigate the operator $L_{\alpha}$, equal to $L+i \alpha T$, where $\alpha \in \mathbb{C}$ (see Remark 2 below and [6, (7.53) p.73] (see also [3] for a detailed study of the operators $L_{\alpha}$ and an explanation of the significance of $L_{\alpha}$ ).

However, we are also motivated by the possibility of explicitly computing the kernel of the Schrödinger propagator $e^{i s L}$, where $s \in \mathbb{R}$, using the analytic continuation of the heat kernel. Indeed, $e^{i s L}$ is the solution operator for the Schrödinger equation

$$
\partial_{s} u(x, y, t, s)=i L u(x, y, t, s)
$$

In [5, p.392-394], Strichartz noticed that "In principle we could attempt to solve (1) by analytic continuation from the solution of the heat equation". However, he abandoned this idea as "this analytic continuation is delicate, so we approach the problem directly". Strichartz proved that, when $s \in \mathbb{R}$, the convolution kernel of the Schrödinger operator $p_{i s}$ is a smooth function on the open set $\left\{(x, y, t) \in \mathbb{H}_{n}(\mathbb{R}):|t|<n|s|\right\}$.

In this note we propose a simple computation which allows us to handle the analytic continuation of the heat kernel in a straightforward manner. Then, using the analytic continuation of the heat kernel we show that $p_{\text {is }}$ is smooth on $\mathbb{H}_{n}(\mathbb{R}) \backslash S_{s}$ when $s \in \mathbb{R}$, and at points in $S_{s}$, the kernel $p_{i s}$ has singularities of Calderon-Zygmund type. This is in contrast to the Euclidean case where, for the standard Laplace operator $\Delta$, the kernel of the operator $e^{i s \Delta}$ is bounded and smooth. In this context it is interesting to note that the convolution kernel of the operator $e^{i s\left(L-T^{2}\right)}$ is smooth. The smoothness of the convolution kernel of the operator $e^{i s\left(L-T^{2}\right)}$ is the last result proved in this note.

A comprehensive discussion of harmonic analysis on the Heisenberg group can be found in [5] or [6, Chapter 1].

The following theorem is the main result of this note.

THEOREM 1. The function $p_{1}$ extends to an analytic function on $\mathbb{H}_{n}(\mathbb{C}) \backslash S$, where $S=\left\{(x, y, t) \in \mathbb{H}_{n}(\mathbb{C}): \pm i t-n-\left(x^{2}+y^{2} / 4\right) \in 2 \mathbb{N}\right\}$. For all $(x, y, t) \in \mathbb{H}_{n}(\mathbb{C}) \backslash S$,

$$
p_{1}(x, y, t)=\frac{1}{2(4 \pi)^{n+1}}(P(x, y, t)+P(x, y,-t)),
$$


where

(2) $\quad P(x, y, t)=2^{n} n ! \sum_{h=0}^{\infty} \sum_{j=0}^{\infty}\left(\begin{array}{c}n+j+h-1 \\ h\end{array}\right)\left(\begin{array}{c}n+j \\ j\end{array}\right) \frac{B^{j}}{(n+2(j+h)-A)^{n+j+1}}$.

The sum (2) is absolutely uniformly convergent on compact subsets of $\mathrm{H}_{n}(\mathbb{C}) \backslash S$. Moreover,

$$
p_{s}(x, y, t)=\frac{1}{s^{n+1}} p_{1}\left(\frac{x}{\sqrt{s}}, \frac{y}{\sqrt{s}}, \frac{t}{s}\right)
$$

for all $s$ such that $\operatorname{Re} s \geqslant 0$.

ProOF: By virtue of the well known formula for the heat kernel on the Heisenberg group (see $[6,(7.36)$, p.71], or $[5,(5.20)]$ )

$$
\begin{aligned}
& 2 p_{1}(x, y, t)= \frac{2}{(4 \pi)^{n+1}} \int_{0}^{\infty} \frac{\lambda^{n} \cos t \lambda}{(\sinh \lambda)^{n}} \exp \left(\frac{-\lambda\left(x^{2}+y^{2}\right) \operatorname{coth} \lambda}{4}\right) \mathrm{d} \lambda \\
&= \frac{1}{(4 \pi)^{n+1}} \int_{0}^{\infty} \frac{\lambda^{n} e^{i t \lambda}}{(\sinh \lambda)^{n}} \exp \left(\frac{-\lambda\left(x^{2}+y^{2}\right) \operatorname{coth} \lambda}{4}\right) \mathrm{d} \lambda \\
& \quad \quad+\frac{1}{(4 \pi)^{n+1}} \int_{0}^{\infty} \frac{\lambda^{n} e^{-i t \lambda}}{(\sinh \lambda)^{n}} \exp \left(\frac{-\lambda\left(x^{2}+y^{2}\right) \operatorname{coth} \lambda}{4}\right) \mathrm{d} \lambda \\
&=\frac{1}{(4 \pi)^{n+1}}(P(x, y, t)+P(x, y,-t)),
\end{aligned}
$$

say. We note that, if $\lambda>0$ and $m \in \mathbb{N}$, then

$$
\left(e^{\lambda}-e^{-\lambda}\right)^{-m}=\sum_{k=0}^{\infty}\left(\begin{array}{c}
m+k-1 \\
k
\end{array}\right) e^{-(2 k+m) \lambda},
$$

where $\left(\begin{array}{c}k-1 \\ k\end{array}\right)=1$ if $k=0$ and $\left(\begin{array}{c}k-1 \\ k\end{array}\right)=0$ otherwise. Now

$$
\begin{aligned}
& 2^{-n} P(x, y, t) \\
& =\int_{0}^{\infty} \sum_{k=0}^{\infty}\left(\begin{array}{c}
n+k-1 \\
k
\end{array}\right) \lambda^{n} e^{i t \lambda} e^{-(2 k+n) \lambda} \exp \left(\frac{\lambda B \operatorname{coth} \lambda}{2}\right) \mathrm{d} \lambda \\
& =\int_{0}^{\infty} \sum_{k=0}^{\infty}\left(\begin{array}{c}
n+k-1 \\
k
\end{array}\right) \lambda^{n} \exp (\lambda(A-2 k-n)) \exp \left(\frac{\lambda B(\operatorname{coth} \lambda-1)}{2}\right) \mathrm{d} \lambda \\
& =\int_{0}^{\infty} \sum_{k=0}^{\infty} \sum_{j=0}^{\infty}\left(\begin{array}{c}
n+k-1 \\
k
\end{array}\right) \lambda^{n} \exp (\lambda(A-2 k-n)) \frac{(\lambda B(\operatorname{coth} \lambda-1))^{j}}{2^{j} j !} \mathrm{d} \lambda \\
& =\int_{0}^{\infty} \sum_{k=0}^{\infty} \sum_{j=0}^{\infty}\left(\begin{array}{c}
n+k-1 \\
k
\end{array}\right) \lambda^{n} \exp (\lambda(A-2 k-j-n)) \frac{(\lambda B)^{j}}{j !\left(e^{\lambda}-e^{-\lambda}\right)^{j}} \mathrm{~d} \lambda
\end{aligned}
$$




$$
\begin{aligned}
& =\int_{0}^{\infty} \sum_{k=0}^{\infty} \sum_{j=0}^{\infty} \sum_{l=0}^{\infty}\left(\begin{array}{c}
n+k-1 \\
k
\end{array}\right)\left(\begin{array}{c}
j+l-1 \\
l
\end{array}\right) \lambda^{n+j} \exp (\lambda(A-n-2(k+j+l))) \frac{B^{j}}{j !} \mathrm{d} \lambda \\
& =n ! \sum_{k=0}^{\infty} \sum_{j=0}^{\infty} \sum_{l=0}^{\infty}\left(\begin{array}{c}
n+k-1 \\
k
\end{array}\right)\left(\begin{array}{c}
j+l-1 \\
l
\end{array}\right)\left(\begin{array}{c}
n+j \\
j
\end{array}\right) \frac{B^{j}}{(n+2(k+j+l)-A)^{n+j+1}} .
\end{aligned}
$$

Note that, for any compact subset $K$ of $\mathbb{H}_{n}(\mathbb{C}) \backslash S$, the sum in the last line of (4) is absolutely uniformly convergent on $K$ and that for all $(x, y, t) \in \mathbb{H}_{n}(\mathbb{R})$ all expressions in the formula (4) are absolutely convergent. Indeed, there exists a constant $C_{K}$ such that

$$
\left|(n+2(k+j+l)-A)^{-n-j-1}\right| \leqslant C_{K}(k+j+l+1)^{-n-j-1} \quad \forall(x, y, t) \in K .
$$

Next,

$$
\left(\begin{array}{c}
n+k-1 \\
k
\end{array}\right) \leqslant C_{n} k^{n-1} \text { and }\left(\begin{array}{c}
j+l-1 \\
l
\end{array}\right) \leqslant(j+k+l+1)^{j-1} / j !
$$

Hence

$$
\begin{aligned}
& \sum_{k=0}^{\infty} \sum_{j=0}^{\infty} \sum_{l=0}^{\infty}\left(\begin{array}{c}
n+k-1 \\
k
\end{array}\right)\left(\begin{array}{c}
j+l-1 \\
l
\end{array}\right)\left(\begin{array}{c}
n+j \\
j
\end{array}\right)\left|\frac{B^{j}}{(n+2(k+j+l)-A)^{n+j+1}}\right| \\
& \leqslant C_{n} C_{n+1} C_{K} \sum_{k=0}^{\infty} \sum_{j=0}^{\infty} \sum_{l=0}^{\infty} \frac{k^{n-1} j^{n}|B|^{j}}{j !(k+j+l+1)^{n+2}} \\
& \leqslant C_{n}^{\prime} C_{K} \sum_{k=0}^{\infty} \sum_{j=0}^{\infty} \frac{k^{n-1} j^{n}|B|^{j}}{j !(k+j+1)^{n+1}} \\
& \leqslant C_{n}^{\prime} C_{K} \sum_{k=0}^{\infty} \sum_{j=0}^{\infty} \frac{|B|^{j} j^{n}}{j !(k+j+1)^{2}} \\
& \leqslant C_{n}^{\prime \prime} C_{K} \sum_{j=0}^{\infty} \frac{|B|^{j} j^{n}}{(j+1) !} \\
& \leqslant C_{K, n} .
\end{aligned}
$$

Now we note that, for any $(x, y, t) \in \mathbb{H}_{n}(\mathbb{R})$,

$$
|(n+2(k+j+l)-A)| \geqslant\left(n+2(k+j+l)-\frac{B}{2}\right),
$$

and $\sup _{\lambda \in \mathbb{R}+} \lambda(\operatorname{coth} \lambda-1)=1$, so

$$
\begin{aligned}
& n ! \sum_{k=0}^{\infty} \sum_{j=0}^{\infty} \sum_{l=0}^{\infty}\left(\begin{array}{c}
n+k-1 \\
k
\end{array}\right)\left(\begin{array}{c}
j+l-1 \\
l
\end{array}\right)\left(\begin{array}{c}
n+j \\
j
\end{array}\right)\left|\frac{B^{j}}{(n+2(k+j+l)-A)^{n+j+1}}\right| \\
& \leqslant n ! \sum_{k=0}^{\infty} \sum_{j=0}^{\infty} \sum_{l=0}^{\infty}\left(\begin{array}{c}
n+k-1 \\
k
\end{array}\right)\left(\begin{array}{c}
j+l-1 \\
l
\end{array}\right)\left(\begin{array}{c}
n+j \\
j
\end{array}\right) \frac{(-B)^{j}}{(n+2(k+j+l)-B / 2)^{n+j+1}}
\end{aligned}
$$




$$
\begin{aligned}
& =\int_{0}^{\infty} \sum_{k=0}^{\infty}\left(\begin{array}{c}
n+k-1 \\
k
\end{array}\right) \lambda^{n} \exp (-\lambda(2 k+n-B / 2)) \exp \left(\frac{-\lambda B(\operatorname{coth} \lambda-1)}{2}\right) \mathrm{d} \lambda \\
& \leqslant 2^{n} \int_{0}^{\infty} \frac{\lambda^{n}}{(\sinh \lambda)^{n}} \exp \left(\frac{B(\lambda-1)}{2}\right) \mathrm{d} \lambda
\end{aligned}
$$

The Lebesgue monotone convergence theorem proves the absolute convergence for all $(x, y, t) \in \mathbb{H}_{n}(\mathbb{R})$.

We obtain (2) by virtue of the identity

$$
\sum_{\substack{k \geqslant 0 \\
l \geqslant 0 \\
k+l=h}}\left(\begin{array}{c}
n+k-1 \\
k
\end{array}\right)\left(\begin{array}{c}
m+l-1 \\
l
\end{array}\right)=\left(\begin{array}{c}
n+m+h-1 \\
h
\end{array}\right)
$$

and (4). To conclude the proof of (3) we note that $L$ is a homogeneous operator so (3) holds when $s>0$ (see [6, Proposition 7.3, p.71]) and both sides of (3) are analytic as a function of $s$ when $\operatorname{Re} s>0$.

COROLlary 2. When $s \in \mathbb{R}$, the convolution kernel of the Schrödinger operator $p_{\text {is }}$ is smooth on $\mathbb{H}_{n}(\mathbb{R}) \backslash S_{s}$, where $S_{s}=\left\{(0,0, \pm s k) \in \mathbb{H}_{n}(\mathbb{R}): k=n, n+2, n+4, \ldots\right\}$. At every point of $S_{s}$, the kernel $p_{i s}$ has a singularity of Calderón-Zygmund type.

Proof: Corollary 2 is a straightforward consequence of (3), and (4) or (2). REMARK 1. There is an alternative proof of Theorem 1. One can use the formula [5, (2.28)] to prove that $P(x, y, t)$ is equal to

$$
2^{n} \sum_{k=0}^{\infty}\left(\frac{(n+k) !}{k !} \frac{(B+(-A+n+2 k))^{k}}{(-A+n+2 k)^{n+1+k}}\right)\left(1+\frac{k}{n+k} \frac{A-n-2 k}{B+(-A+n+2 k)}\right) .
$$

An argument similar to that in the proof of Theorem 1 shows that the above sum is uniformly absolutely convergent on any compact subset of $\mathbb{H}_{n}(\mathbb{C}) \backslash S$.

REmarK 2. In [6, (7.53), p.73], Taylor noticed that, when $s>0$ and $|\operatorname{Re} \alpha|<n$,

$$
K_{e^{-s L_{\alpha}}}(x, y, t)=p_{s}(x, y, t-i s \alpha),
$$

where $K_{e^{-s L_{\alpha}}}$ is the convolution kernel of the operator $e^{-s L_{\alpha}}$ and $L_{\alpha}=L+i \alpha T$. By virtue of Theorem 1, (5) holds also when $|\operatorname{Re} \alpha|=n$. Note that, when $|\operatorname{Re} \alpha|=n$, the kernel $K_{e^{-s L_{\alpha}}}, s>0$ is no longer smooth and it has a singularity of Calderón-Zygmund type at the point $(0,0, s \operatorname{Im} \alpha)$.

We would like to end with another observation, concerning a full Laplace operator on the Heisenberg group. We define this operator by the following formula

$$
\tilde{L}=-\sum_{r=1}^{n} X_{r}^{2}+Y_{r}^{2}-T^{2}=L-T^{2}
$$


TheOREM 3. For any $s \in \mathbb{R} \backslash\{0\}$, the convolution kernel of the operator $e^{-i s \tilde{L}}$ extends to an analytic function on $\mathbb{H}_{n}(\mathbb{C})$.

Proof: Write $\gamma$ for $e^{i \pi / 4}$. We denote the convolution kernels of the operators $e^{-i s \tilde{L}}$ and $e^{-s\left(\gamma L-T^{2}\right)}$ by $K_{e^{-i s \bar{L}}}$ and $K_{e^{-s\left(\gamma L-r^{2}\right)}}$ respectively. Note that (see [6, (7.36)] and (3))

$$
K_{e^{-s\left(\gamma L+T^{2}\right)}}(x, y, t)=\frac{1}{(4 \pi \gamma)^{n+1}} \int_{0}^{\infty} \frac{\lambda^{n} \cos (t \lambda /(\gamma s))}{(\sinh \lambda)^{n}} \exp \left(\frac{-\lambda\left(x^{2}+y^{2}\right) \operatorname{coth} \lambda}{4 \gamma s}\right) e^{-s \lambda^{2}} \mathrm{~d} \lambda .
$$

Hence $K_{e^{-s\left(\gamma L+T^{2}\right)}}$ is an analytic function on $\mathbb{H}_{n}(\mathbb{C})$. Now to finish the proof it is enough to note that

$$
K_{e^{-i s \tilde{L}}}(x, y, t)=K_{e^{-s\left(\gamma \cdot \gamma L+\gamma^{2} T^{2}\right)}}(x, y, t)=\frac{1}{\gamma^{n+1}} K_{e^{-s\left(\gamma L+T^{2}\right)}}\left(\frac{x}{\sqrt{\gamma}}, \frac{y}{\sqrt{\gamma}}, \frac{t}{\gamma}\right)
$$

for all $s>0$ and $(x, y, t) \in \mathbb{H}_{n}(\mathbb{R})$ (see $[4,(18)]$ ).

REMARK 3. When Re $s>0$, one can use the theory of analytic hypoellipticity to investigate the smoothness and analyticity of the kernel $K_{e^{-s \tilde{L}}}$ see, for example, $[1,2]$. However, analytic hypoellipticity cannot be used directly to investigate the convolution kernel of $K_{e^{-s \tilde{L}}}$ when $\operatorname{Re} s=0$.

\section{REFERENCES}

[1] M. Christ, 'Analytic hypoellipticity, representations of nilpotent groups, and a nonlinear eigenvalue problem', Duke Math. J. 72 (1993), 595-639.

[2] M. Christ, 'Nonexistence of invariant analytic hypoelliptic differential operators on nilpotent groups of step greater than two', in Essays on Fourier analysis in honor of Elias M. Stein (Princeton, NJ, 1991), Princeton Math. Series 42 (Princeton Univ. Press, Princeton, NJ, 1995), pp. 127-145.

[3] G.B. Folland and E.M. Stein, 'Estimates for the $\bar{\partial}_{b}$ complex and analysis on theHeisenberg group', Comm. Pure Appl. Math. 27 (1974), 429-522.

[4] A. Sikora, 'On the $L^{2} \rightarrow L^{\infty}$ norms of spectral multipliers of "quasi-homogeneous" operators on homogeneous groups', Trans. Amer. Math. Soc. 351 (1999), 3743-3755.

[5] R.S. Strichartz, ' $L^{p}$ harmonic analysis and Radon transforms on the Heisenberg group', $J$. Funct. Anal. 96 (1991), 350-406.

[6] M.E. Taylor, Noncommutative harmonic analysis, Mathematical Surveys and Monographs 22 (American Mathematical Society, Providence, R.I., 1986).

Department of Mathematical Sciences

New Mexico State University

PO Box 30001

Las Cruces NM 88003

Untied States of America

e-mail: asikora@nmsu.edu
Instytut Matematyczny

Uniwersytet Wroclawski

50-384 Wroclaw

pl. Grunwaldzki 2/4

Poland

e-mail: zenek@math.uni.wroc.pl 